\title{
MR-mammography - impact on disease extent determination and surgical treatment of invasive ductal and lobular breast cancers
}

\author{
V. LEHOTSKA*, K. RAUOVA, L. VANOVCANOVA \\ 2nd Radiology Clinic, St. Elisabeth's Cancer Institute Medical Faculty Comenius University, Comenius University, Bratislava, Slovakia \\ ${ }^{*}$ Correspondence: viera.lehotska@ousa.sk
}

Received March 12, 2014 / Accepted June 24, 2014

\begin{abstract}
The aim of this study is to assess the impact of multiparametric MR imaging to an exact determination of the extent of invasive ductal and lobular breast cancers in routine clinical practice.180 women aged 27-74 years (median 52.4 years) with newly diagnosed invasive ductal and lobular breast cancers confirmed by core-cut or vacuum-assisted biopsy were examined by all three imaging modalities, i.e. digital X-ray mammography, ultrasonography and 3T MR-mammography. In case of MR-mammography an extended protocol, i.e. combination of morphological breast MRI + DCE + DWI was used. For overall detection and determination of the extent of invasive breast cancers (IDC + ILC) MRmammography alone reached the diagnostic accuracy of $72.48 \%$, while X-ray mammography $69.12 \%$ and ultrasonography $59.87 \%$. In cases of ILC combination of X-ray mammography and MR- mammography with sensitivity of $96.15 \%$ was the most effective. In comparison with X-ray mammography and ultrasonography MR-mammography had higher sensitivity $(96.15 \%$, versus 90.28\%) in the diagnosis of multifocal/ multicentric invasive lobular carcinoma and invasive ductal carcinoma with extensive intraductal component, as well. 3T MR-mammography is an effective complementary consulting modality to digital X-ray mammography and ultrasonography, and it is particularly beneficial in the detection of additional mammographically and ultrasonografically occult breast lesions, as well as in the determination of the real extent of pathological changes in the ipsilateral and contralateral breast.
\end{abstract}

Key words: MR-mammography, occult breast cancer, invasive ductal cancer, invasive lobular cancer, multifocality, multicentricity

Detection and diagnosis of breast lesions are primarily based on the X-ray mammography, both in group of asymptomatic women in screening program as well as in women with palpation findings. The main goal is to find any changes, try to determine their extent and to assess local spread. The sensitivity of X-ray mammography varies according to technical parameters, physician competence and measurement criteria, and is reported between $69 \%$ and $90 \%$ [1]. Furthermore, X-ray mammography (MG) and ultrasonography (US) is limited by soft tissue contrast and the ability to measure tumor vascularity. Women with suspicious X-ray mammography are referred for additional targeted mammography examinations and breast ultrasound, followed by percutaneous biopsy. Approximately in $10-20 \%$ of patients with benign changes in preoperative histology are found one or more occult malignant lesions in the definitive histology of surgically retrieved tissue [2,
$3,4]$. Those lesions cause morphological and functional changes in the MR image, but do not correlate with mammographical and ultrasonographical images. The potential of MR - mammography currently lies in the high sensitivity of dynamic contrast-enhanced images (DCE) for the detection of malignant breast tumors (92-99\%) [5, 6]. Since only a few studies correlate histopathological findings with the extent and nature of changes in the MR image, this method is still not standardly used in clinical practice. Also, no study has confirmed the potential of advanced breast MRI protocol at 3 Tesla MR device for detecting and determining the extent of breast cancer. The final product of this study is to highlight the benefits of multiparametric breast MR imaging, which being correlated with histopathological changes allows more exact determination of the nature and extent of pathological process in the breast and contributes to effective planning of breast surgery. 


\section{Patients and methods}

Patients were selected from the total number of 575 women, who underwent diagnostic breast imaging and had histologically proven pathological breast changes. We excluded 114 patients with histologically confirmed DCIS and 281 patients with a solitary breast lesion. These patients were analyzed separately and the analysis is out of the scope of this article.

Remaining 180 women, aged 27-74 years (median 52.4 years) with multiple breast lesions (total number of lesion 476) were included in this study, out of which 326 (68.49\%) were newly diagnosed with invasive ductal and lobular breast cancers. The patients were examined by all three imaging modalities, i.e. digital X-ray mammography (Lorad Dimensions, Hologic, USA, 2010), ultrasound (iU22 Philips, Belgium, 2010) and 3T MR-mammography with an extended protocol, i.e. combination of morphological breast MRI + DCE + DWI (3T Magnetom Verio, Siemens, Belgium, 2010).

When evaluating MR-mammography, we focused on the following facts:

- number and location of lesions,

- changes in morphological characteristics: signal intensity

(SI) on T2-weighted image, shape and margins of the lesion, homogeneity of contrast enhancement, presence of peripheral and/or ductal enhancement,

- pharmacokinetics of gadolinium in the lesion, and

- the presence of restricted diffusion in the ADC map.

As basic postprocessing we used postcontrast subtraction images, perfusion map, wash- in and wash- out maps, TTP map ("time - to-peak") and ADC map for DWI. We generated kinetic curves (TICs) of the lesions and evaluated their character depending on the percentage signal intensity increase and on the shape of the kinetic curve.

We used multiparametric evaluation of each MRI differentiable lesion while following parameters were assessed: SI in T2-weighted images, morphological and functional characteristics in DCE-study, and subsequently SI of the lesion in DWI, and presence of diffusion restriction in ADC map.

In the DCE-study as key functional signs of malignancy we considered: irregular lesions margins, stellate or spicular shape, peripheral ("ring") enhancement, pathological ductal enhancement - isolated, branching out in segmental, regional or diffuse distribution and shape of kinetic curves (continuous increase - TIC type 1, increase with "plateau" - TIC type 2, increase with wash- out - TIC type 3 ) .

Based on the presence or absence of these signs, we categorized the lesion as suspicious for malignancy or as benign one. MRI findings were compared with mammography and/ or ultrasound findings, and we took into account the number of corresponding MRI, MG and US changes. If we found MRI changes, which not correlate with the X-ray mammography and / or ultrasound images, we recommended to perform targeted "second-look" ultrasound examination. All additional lesions found in "second-look" ultrasound were subsequently biopted. If any MRI differentiable lesion was occult in targeted ultrasound examination, we performed vacuum-assisted biopsy (VAB) of that lesion under MRI guidance. In the case of multiple lesions in one breast or both breasts, a targeted biopsy was performed of no more than three lesions on each side, taking into account the distribution of lesions in breast quadrants. If there were two focuses in the breast, then both were verified by biopsy regardless of their location. Samples, which were obtained during interventional procedures (core- biopsy, VAB), were fixed in formaldehyd. Specimens were evaluated by experienced histopathologists, who categorized changes as benign, "high risk" precursor lesions, ductal carcinoma in situ (DCIS) and invasive cancer - ductal (IDC), ductal with extensive intraductal component (IDC with EIC), and lobular (ILC). In addition to determining the histological type of lesion the immunohistochemical profile of invasive cancer and the grade of nuclear atypia in the case of DCIS were evaluated. An extensive intraductal component (EIC) was defined as tumor with an invasive component, where at least $25 \%$ of the tumor was DCIS and there were additional discrete foci of DCIS outside the main tumor mass [7, 8].

Statistical methods. In the statistical evaluation of results, we used the "golden standard" - correlation of imaging findings with histopathological findings. To identify the relationship between independent parameters (SI in T2-weighted image, shape and margins of the lesion, postcontrast enhancement kinetics, SI in DWI and ADC maps) and the final diagnosis (benign and malignant breast lesions), we used logistic regression modeling. When analyzing results in order to determine the diagnostic accuracy of each modality, we considered as truly positive finding (P), if the lesion with biopsy proven malignancy was also considered as the lesion suspicious of malignancy in that particular modality. Lesions, which were in MG, ultrasound and MRI identified as benign, but histology proved carcinoma, were classified as false negative (FN) in the final assessment. Similarly, in cases where the lesion was confirmed only by one modality (e.g. MR-mammography followed by the finding of the "second-look" ultrasound) findings in all remaining modalities we considered as false negative. If according of any modality (respectively by supplementary MR-mammography) we performed an additional biopsy, which was histologically proven as a bening, we considered the finding of that particular modality as false positive (FP). The sensitivity and accuracy of MR-mammography in the determination of disease extent for invasive breast cancers (IDC, IDC with EIC and ILC) were compared with the sensitivity and accuracy of X-ray mammography and ultrasonography using the chi-squared test to determine statistical significance (StatCalc, version 7.1.2, Excel 2010). The level of statistical significance was determined as $\mathrm{P}<0.05$. Logistic regression analysis was performed using software MedCalc ${ }^{\circledR}$, version 12.4.0, 2003-2013. 


\section{Results}

The study includes 180 women with a total of 476 breast lesions, of which 326 (68.49\%) were malignant. Of these IDC was confirmed in $61.96 \%(n=202)$, IDC with extensive intraductal component (EIC) in $22.09 \%(\mathrm{n}=72)$ and ILC in $15.95 \%$ $(\mathrm{n}=52) .23$ out of 150 remaining histologically benign breast lesions showed signs of atypia: 2 papillomas, 4 radial sclerosing lesions, 3 atypical lobular hyperplasias ( 1 associated with microfocus of LCIS) and 14 atypical ductal hyperplasias.

The combination of X-ray mammography and MRmammography (322 of 326) provides the highest sensitivity

Table 1. Summary of $\mathbf{4 7 6}$ histologically proven breast lesions according to the method of determination

\begin{tabular}{|c|c|}
\hline Histology + Modality & Number of Lesions (\%) \\
\hline \multicolumn{2}{|l|}{ Malignant breast lesions $(n=326)$} \\
\hline Digital X-ray Mammography & $239 / 326(73.31 \%)$ \\
\hline Ultrasonography & $273 / 326(83.74 \%)$ \\
\hline X-ray Mammography + Ultrasonography & $299 / 326(91.72 \%)$ \\
\hline MR-Mammography & $307 / 326(94.17 \%)$ \\
\hline X-ray Mammography + MR-Mammography & $322 / 326(98.77 \%)$ \\
\hline \multicolumn{2}{|l|}{$I D C(n=202)$} \\
\hline Digital X-ray Mammography & $184 / 202(91.09 \%)$ \\
\hline Ultrasonography & $189 / 202(93.56 \%)$ \\
\hline X-ray Mammography + Ultrasonography & $195 / 202(96.5 \%)$ \\
\hline MR-Mammography & $191 / 202(94.55 \%)$ \\
\hline X-ray Mammography + MR-Mammography & $202 / 202(100 \%)$ \\
\hline \multicolumn{2}{|l|}{ IDC with EIC $(n=72)$} \\
\hline Digital X-ray Mammography & $42 / 72(58.33 \%)$ \\
\hline Ultrasonography & $38 / 72(52.78 \%)$ \\
\hline X-ray Mammography + Ultrasonography & $54 / 72(75 \%)$ \\
\hline MR-Mammography & $65 / 72(90.28 \%)$ \\
\hline X-ray Mammography + MR-Mammography & $72 / 72(100 \%)$ \\
\hline \multicolumn{2}{|l|}{$I L C(n=52)$} \\
\hline Digital X-ray Mammography & $25 / 52(48.08 \%)$ \\
\hline Ultrasonography & $45 / 52(86.54 \%)$ \\
\hline X-ray Mammography + Ultrasonography & $45 / 52(86.54 \%)$ \\
\hline MR-Mammography & $50 / 52(96.15 \%)$ \\
\hline X-ray Mammography + MR-Mammography & $50 / 52(96.15 \%)$ \\
\hline \multicolumn{2}{|l|}{ Benign breast lesions with atypia $(n=23)$} \\
\hline Digital X-ray Mammography & $13 / 23(56.52 \%)$ \\
\hline Ultrasonography & $21 / 23(91.30 \%)$ \\
\hline X-ray mammography + Ultrasonography & $22 / 23(95.65 \%)$ \\
\hline MR-Mammography & $21 / 23(91.30 \%)$ \\
\hline X-ray Mammography + MR-Mammography & $22 / 23(95.65 \%)$ \\
\hline \multicolumn{2}{|l|}{ Benign breast lesions without atypia $(n=127)$} \\
\hline Digital X-ray Mammography & $47 / 127(37.0 \%)$ \\
\hline Ultrasonography & $87 / 127(68.50 \%)$ \\
\hline X-ray Mammography + Ultrasonography & $96 / 127(75.59 \%)$ \\
\hline MR-Mammography & $91 / 127(71.65 \%)$ \\
\hline X-ray Mammography + MR-Mammography & $118 / 127(92.91 \%)$ \\
\hline
\end{tabular}

$(98.77 \%)$ in detection of malignant mammary lesions (Table 1). Compared with sensitivity of the combination of X-ray mammography and ultrasound $(91.72 \%)$ we observed a statistically significant difference in favor of $\mathrm{MRI}+\mathrm{MG}(\mathrm{p}=$ 0.0003 ) (Table 4). MR-mammography alone, with sensitivity $94.17 \%$ for the diagnosis of malignant mammary lesions, was more successful when compared to the X-ray mammography and ultrasound ( $p<0.0001)$ (Table 2, 3). Statistically the most significant difference compared with the X-ray mammography and ultrasound achieved MR-mammography in the diagnosis of IDC with EIC ( $\mathrm{p}<0.0001)$ (Table 2,3) and when compared with the X-ray mammography alone in the diagnosis of ILC $(\mathrm{p}<0.0001)$ (Table 2).

MR-mammography correctly indicated 191 out of 202 biopsy proven IDC lesions (94.55\%), while the X-ray mammography correlated only 184 of $202(91.09 \%)$ and ultrasonography 189 of 202 (93.56\%). MRI diagnosed 17 out of 18 mammographically occult IDC lesions, of which 15 were later detected by targeted second-look ultrasound examination. Detection of IDC lesions in X -ray mammography was apparently influenced by mammographical density of the breast tissue and the absence of microcalcifications. Conversely, $11 \mathrm{MRI}$ occult IDC lesions were small ( $<5 \mathrm{~mm})$, and were merged with hormone dependent changes. 9 out of them contained fine microcalcifications. Another 6 IDC lesions identified by MR-mammography had no clear correlate in the ultrasound image.

Table 2. Pairwise comparison of digital X-ray mammography and MRmammography, depending on the number of diagnosed histologically confirmed breast lesions

\begin{tabular}{|c|c|c|c|}
\hline Modality & MG & MR & $p$ \\
\hline $\operatorname{IDC}(\mathrm{n}=202)$ & $184 / 202$ & $191 / 202$ & 0.1814 \\
\hline IDC with EIC $(n=72)$ & $42 / 72$ & $65 / 72$ & $<0.0001$ \\
\hline $\operatorname{ILC}(\mathrm{n}=52)$ & $25 / 52$ & $50 / 52$ & $<0.0001$ \\
\hline Benign breast lesions with atypia $(\mathrm{n}=23)$ & $13 / 23$ & $21 / 23$ & 0.0141 \\
\hline Benign breast lesions without atypia $(\mathrm{n}=127)$ & $47 / 127$ & $91 / 127$ & $<0.0001$ \\
\hline All malignant breast lesions $(\mathrm{n}=236)$ & $239 / 326$ & $307 / 326$ & $<0.0001$ \\
\hline
\end{tabular}

Table 3. Pairwise comparison of ultrasonography and MR-mammography based on the number of diagnosed histologically confirmed breast lesions

\begin{tabular}{|c|c|c|c|}
\hline Modality & US & MR & $p$ \\
\hline $\operatorname{IDC}(n=202)$ & $189 / 202$ & $191 / 202$ & 0.0771 \\
\hline IDC with EIC $(\mathrm{n}=72)$ & $38 / 72$ & $65 / 72$ & $<0.0001$ \\
\hline $\operatorname{ILC}(\mathrm{n}=52)$ & $45 / 52$ & $50 / 52$ & 0.1008 \\
\hline Benign breast lesions with atypia $(n=23)$ & $21 / 23$ & $21 / 23$ & 1.0000 \\
\hline Benign breast lesions without atypia $(\mathrm{n}=127)$ & $87 / 127$ & $91 / 127$ & 0.5838 \\
\hline All malignant breast lesions $(n=236)$ & $273 / 326$ & $307 / 326$ & $<0.0001$ \\
\hline
\end{tabular}


Table 4. Pairwise comparison of combinations $\mathrm{X}$-ray mammography and MR-mammography and X-ray mammography and ultrasonography based on the number of diagnosed histologically confirmed breast lesions

\begin{tabular}{|c|c|c|c|}
\hline Histology & $\begin{array}{c}\mathrm{MG}+ \\
\mathrm{MR}\end{array}$ & $\begin{array}{c}\text { MG + } \\
\text { US }\end{array}$ & $p$ \\
\hline $\operatorname{IDC}(n=202)$ & $202 / 202$ & $195 / 202$ & 0.0610 \\
\hline IDC with EIC $(n=72)$ & $72 / 72$ & $54 / 72$ & 0.0070 \\
\hline $\operatorname{ILC}(n=52)$ & $50 / 52$ & $45 / 52$ & 0.1008 \\
\hline Benign breast lesions with atypia $(n=23)$ & $22 / 23$ & $22 / 23$ & 1.0000 \\
\hline Benign breast lesions without atypia $(n=127)$ & $118 / 127$ & $96 / 127$ & 0.0003 \\
\hline All malignant breast lesions $(n=236)$ & $322 / 326$ & $299 / 326$ & 0.0003 \\
\hline
\end{tabular}

MR-mammography (with known X-ray mammography and US image) was proved to be the most reliable method in the determination of real extent of IDC with extensive intraductal component, whereas it displayed and specified all 23 mammographically hidden or underestimated lesions and 29 out of 34 ultrasonographically undervalued lesions ( $\mathrm{p}<0.0001)$. (Table 5).

Overall there were 72 histologically confirmed IDCs with EIC, where EIC was formed by at least $25 \%$ of DCIS or by surrounding discrete foci of DCIS near the invasive component of the tumor.

MR - mammography showed sensitivity of $90.28 \%$ for determining the extent of IDC with EIC (Fig.1), compared with separately applied MG and USG (58.33\% and 52.78\%, respectively; $\mathrm{p}<0.0001)$. Six IDCs with EIC component were displayed only in MR - mammography. If compared with sensitivity of combination of X-ray mammography and MRI examinations and ultrasound alone to determine the actual extent of the IDC with EIC, we achieved a statistically significant difference at probability level $p=0.0191$ and pairwise comparison with a combination of US + MG and MG + MRI $\mathrm{p}=0.0070 . \mathrm{MR}-$ mammography underestimated 7
IDC with EIC, which extent was correctly identified by X-ray mammography (7 of 7) and $57.14 \%$ (4 of 7) by ultrasonography (Table 5).

The sensitivity of MRI for the diagnosis of invasive lobular carcinoma was $96.15 \%$ (Fig.2), and the sensitivity of X-ray mammography and ultrasonography were $48.08 \%$ and $86.54 \%$ respectively. For pairwise comparison of X-ray mammography and MRI in the diagnosis of ILC the statistical significance was $\mathrm{p}<0.0001$, however, we did not confirm the statistical significance between the breast ultrasound and MRI ( $\mathrm{p}=0.1008)$ (Table 3).

X-ray mammography revealed 25 out of 52 ILC lesions, breast ultrasound 45 out of 52, and magnetic resonance imaging 50 out of 52 ILC foci, while 5 ILCs were confirmed only by MR-mammography. Combined imaging by X-ray mammography and ultrasonography detected 45 out of 52 ILCs $(86.54 \%)$ and a combination of MRI and X-ray mammography 50 out of 52 ILC (96.15\%), which was statistically not significant $(\mathrm{p}=0.1008)$. Based on MR-mammography 2 invasive lobular cancers with morphological and functional character of benign mammary lesion were underestimated.

In MR imaging we missed 20 out of 326 malignant breast lesions $(6.13 \%)$, in ultrasound $54(16.56 \%)$ and in X-ray mammography 68 out of 326 cancers (20.86\%) (Table 5). The diagnostic accuracy for assessing the number and range of focal changes of MR-mammography alone reached $72.48 \%$ (Table 6).

Compared with X-ray mammography, MR-mammography diagnosed 66 (20.24\%) additional malignant lesions out of total number of 326 histologically confirmed malignant breast lesions. Compared with ultrasonography there were 40 (12.27\%) other malignant foci. Based on the "second-look" ultrasound we have confirmed 48 (14.72\%) additional lessions out of the total 326 cancers and by subsequent reassessment of X-ray mammography images we found 27 (8.28\%) mammographically differentiable changes. Out of the 106 (32.51\%) occult

Table 5. Overview of numbers of true positive findings and number of occult (unrecognized, hidden) breast lesions based on individual imaging modality and histopathology of malignant lesion

\begin{tabular}{|c|c|c|c|c|}
\hline $\begin{array}{l}\text { Histopathology and Imaging } \\
\text { modality }\end{array}$ & $\begin{array}{l}\text { True positive findings (P) } \\
\text { (\%) }\end{array}$ & $\begin{array}{c}\text { Occult lesions in MG } \\
(\%)\end{array}$ & $\begin{array}{l}\text { Occult lesions in US } \\
\text { (\%) }\end{array}$ & $\begin{array}{l}\text { Occult lesions in MR } \\
\text { (\%) }\end{array}$ \\
\hline IDC $(n=202)$ & & 18 & 13 & 11 \\
\hline X-ray Mammography & $184(91.09)$ & - & $7(53.85)$ & $9(81.81)$ \\
\hline Ultrasonography & $189(93.56)$ & $15(83.33)$ & - & $4(36.36)$ \\
\hline MR-Mammography & $191(94.55)$ & $17(94.44)$ & $6(46.15)$ & - \\
\hline IDC with EIC $(n=72)$ & & 23 & 34 & 7 \\
\hline X-ray Mammography & $42(58.33)$ & - & $20(58.82)$ & $7(100)$ \\
\hline Ultrasonography & $38(52.78)$ & $12(52.17)$ & - & $4(57.14)$ \\
\hline MR-Mammography & $65(90.28)$ & $23(100)$ & $29(85.29)$ & - \\
\hline $\operatorname{ILC}(n=52)$ & & 27 & 7 & 2 \\
\hline X-ray Mammography & $25(48.08)$ & - & 0 & 0 \\
\hline Ultrasonography & $45(86.54)$ & $21(77.77)$ & - & 0 \\
\hline MR-Mammography & $50(96.15)$ & $26(96.30)$ & $5(71.43)$ & - \\
\hline
\end{tabular}


Table 6. Diagnostic performance in 476 histologically proven breast lesions (326 malignant and 150 benign lesions)

\begin{tabular}{lcccc}
\hline Modality & Sensitivity (\%) & Specificity (\%) & Positive Predictive Value (\%) & Accuracy (\%) \\
\hline MG & $239 / 326(73.31)$ & $90 / 150(60)$ & $239 / 299(79.93)$ & $329 / 476(69.12)$ \\
US & $273 / 326(83.74)$ & $42 / 150(28)$ & $273 / 381(71.65)$ & $285 / 476(59.87)$ \\
MG + US & $299 / 326(91.72)$ & $32 / 150(21.33)$ & $299 / 417(71.70)$ & $331 / 476(69.54)$ \\
MRI & $307 / 326(94.17)$ & $38 / 150(25.33)$ & $307 / 419(73.27)$ & $345 / 476(72.48)$ \\
MG + MRI & $322 / 326(98.77)$ & $10 / 150(6.66)$ & $322 / 462(69.70)$ & $332 / 476(69.75)$ \\
\hline
\end{tabular}
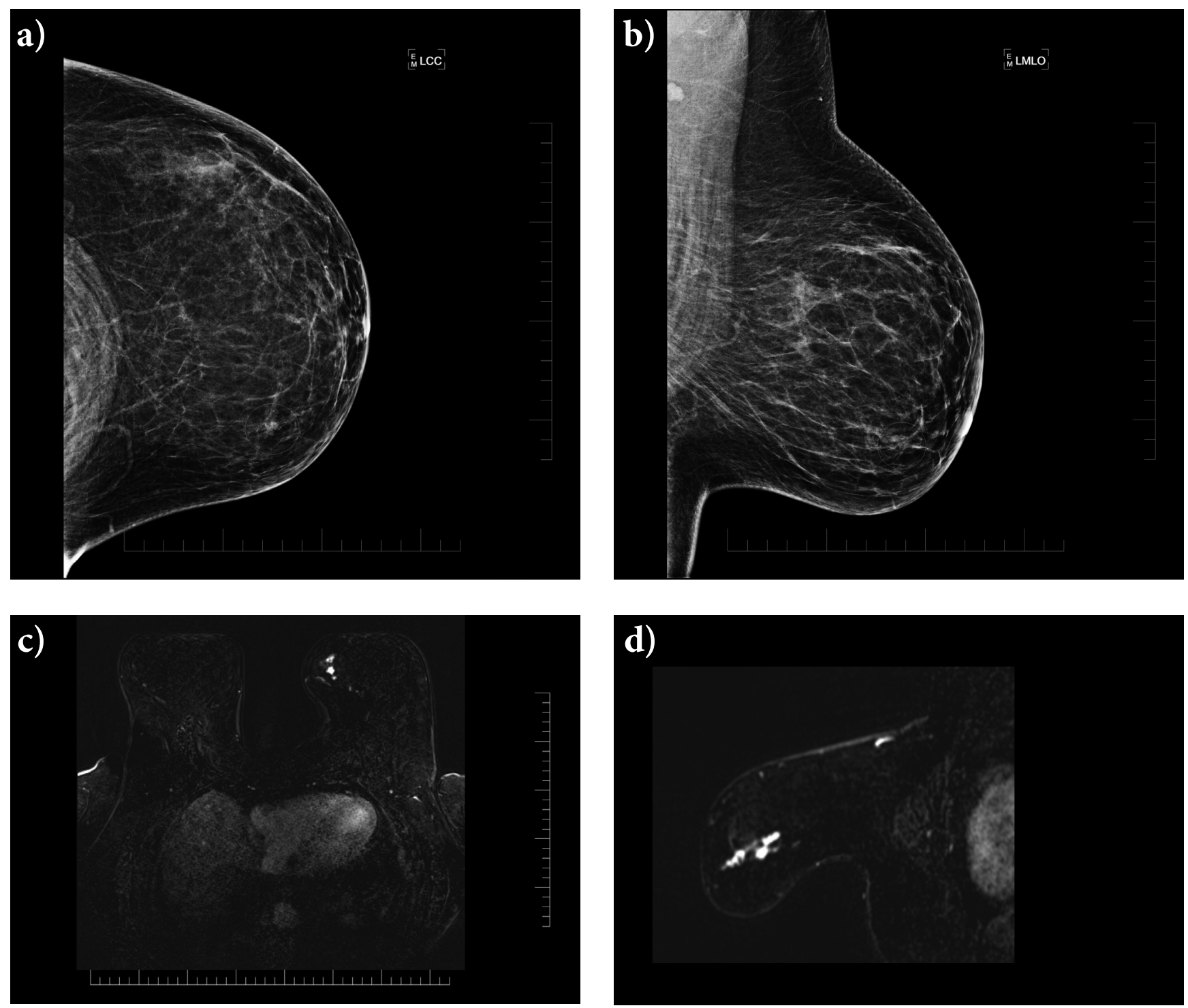

Figure 1. Images of 57-year old woman with invasive ductal carcinoma (IDC) with extensive intraductal component (EIC) differentiated only at MRmammography.

a) Craniocaudal (CC) and b) mediolateral oblique (MLO) views show 8-mm irregular condensation with microcalcifications in the lower inner part of the left breast (circle). Vacuum-assisted biopsy under stereotactic $\mathrm{X}$-ray guidance revealed IDC.

c) Transverse subtracted T1- weighted flash $3 \mathrm{D}$ MR acquisition obtained 20 seconds after intravenous administration of $0.1 \mathrm{mmol} / \mathrm{kg}$ gadopentetate dimeglumine (flip angle, $45^{\circ}$; section thickness, $1 \mathrm{~mm}$; field of view, $30 \mathrm{~cm} ; 3.0 \mathrm{~T}$ ) and sagital MIP reconstruction d) shows intense $42 \mathrm{~mm}$ linear clumped enhancement in lower inner left breast extending posteriorly and anteriorly to the known invasive cancer, due to extensive intraductal component (EIC). Patient underwent breast conserving surgery after stereotactic-guided needle localization and postoperative histopathology confirmed IDC with intraductal carcinoma (EIC) of total extent $43 \mathrm{~mm}$. 


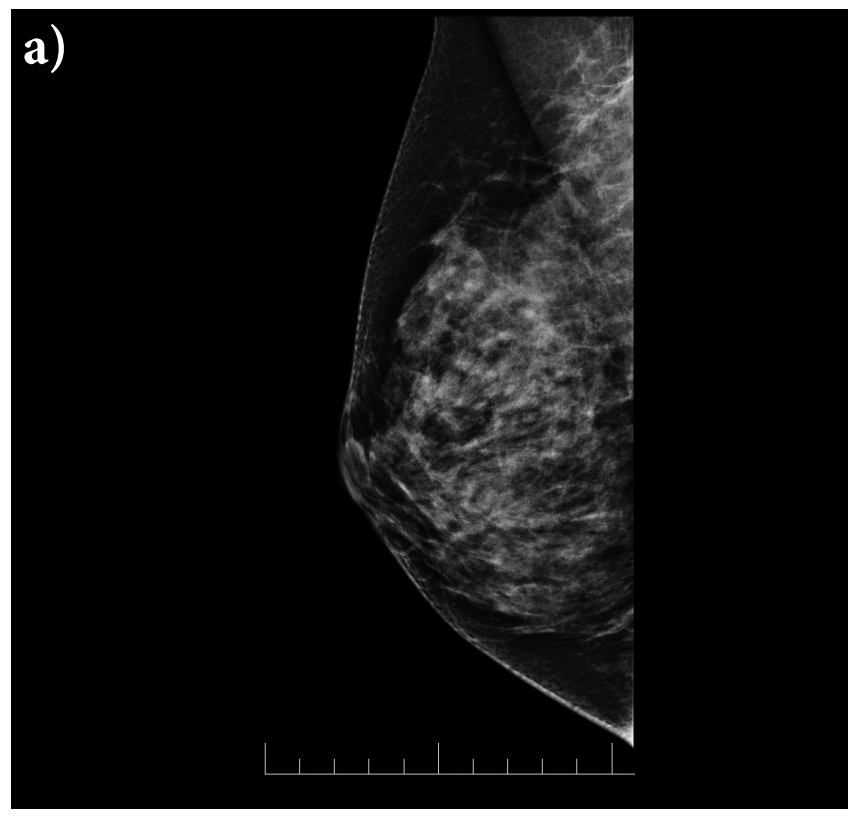

b)

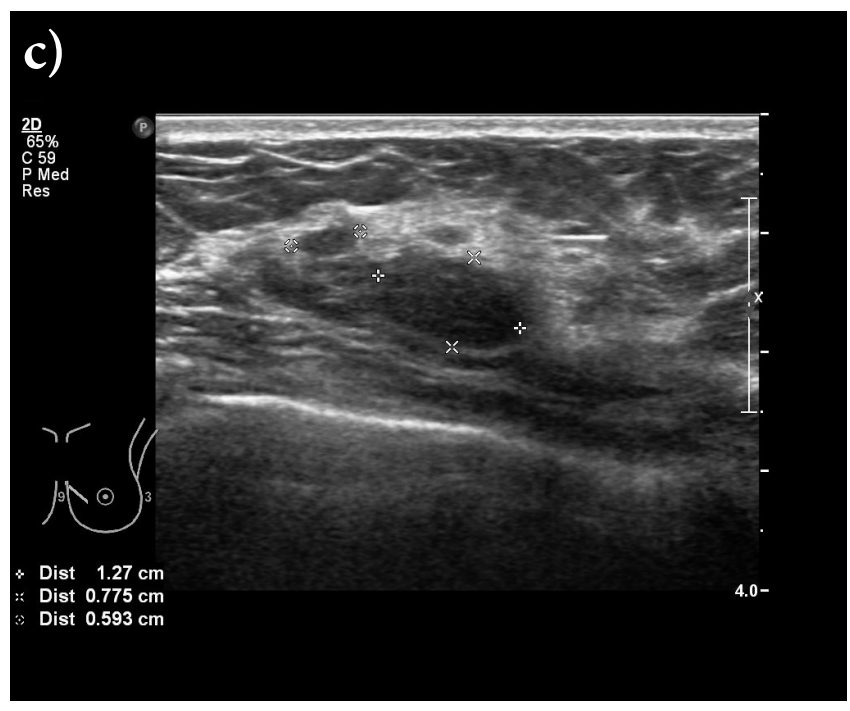

d)
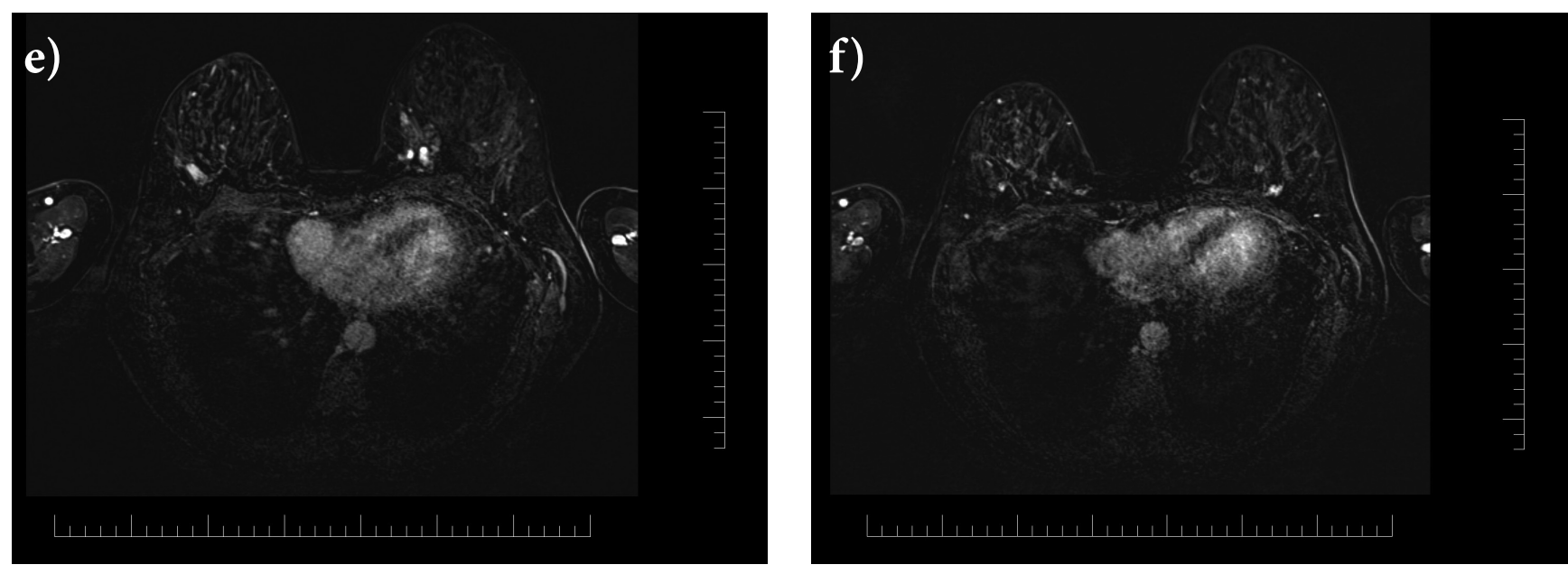


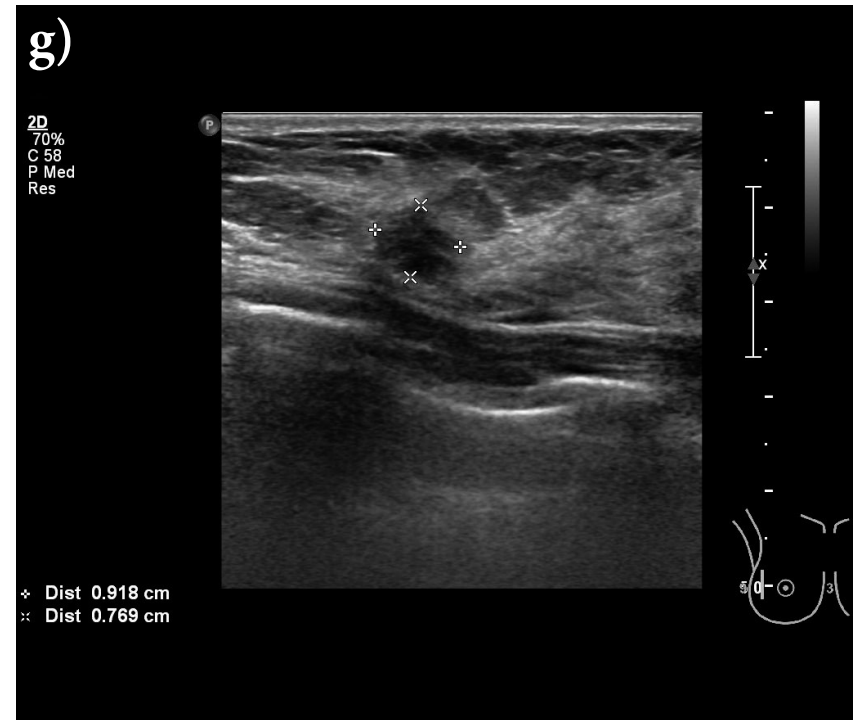

or underestimated changes $75(23 \%)$ lesions were proved as malignants. The remaining 31 (9.51\%) malignant breast lesions were visible only in the MR image.

\section{Discussion}

The main goal of breast imaging is early detection of focal changes, which are suspected of malignant transformation. During more than 30 years of X-ray mammography screening in the developed countries of Europe and worldwide it is clearly confirmed that X-ray mammography is an effective method of early breast cancer diagnosis in group of women aged 50 and more. In some countries, screening for breast cancer starts at the age of 40 . In dense or heterogeneously dense breast the sensitivity of X-ray mammography for the detection of malignant focal changes is considerably lower. Studies of Mandelson M.T. et al. (2000) and T. M. Kolb et al. (2002) reported lowest sensitivity (30 to $48 \%$ ) of X-ray mammography for diagnosis of breast cancer in extremely dense breast and $70 \%$ in heterogeneously dense breast, while the sensitivity of X-ray mammography for the detection of ILC is lower than the sensitivity for detection of IDC $[9,10,11,12]$. Same studies confirmed higher prevalence of cancer $(62.7 \%)$ in dense and heterogeneously dense fibroglandular tissue $[9,10]$. The presence of mammographically occult breast lesions, especially in dense breast in younger women and in women with delayed involution led to the observation that in these women were more often found inadequate or positive resection margins after breast conserving surgery, as well as higher risk for developing local recurrence. This fact led to widespread use of complementary imaging techniques such as ultrasound and in the last 20 years increasing use of magnetic resonance imaging, which clarifies the extent of pathological changes in the breast as well as the presence of mammographically occult lesions $[11,12]$.
Figure 2. Images of 47-year-old woman with bilateral ILC.

a), b) Bilateral mediolateral oblique (MLO) X-ray mammography shows heterogeneously dense breast parenchyma with several opacities and condensations.

c) Ultrasound image ( $\mathrm{L} 12.5 \mathrm{MHz}$ transducer) obtained in 9-10 o'clock position shows multiple hypoechoic lesions in the left breast (landmarks).

d), e) and f) Transverse subtracted MR images obtained 2 minutes after contrast agent injection (same parameters as in Fig. 1) shows multiple enhancing foci in both breasts, suggesting multifocality and multicentricity of the process.

g) Second-look US image (L 12.5 MHz transducer) obtained in 9 o'clock position in right breast demonstrates hypoechoic mass (landmarks). Both US visible masses underwent core-needle biopsy with US guidance, confirming ILC.

Patient underwent bilateral mastectomy which confirmed diffuse ILC.

Diagnosis of ILC still remains a problem, while it might be subtle in the X-ray mammography image and therefore can be easily overlooked. In case of ILC ultrasonography has a good potential to differentiate asymmetric mammographical density or structure disorders, but due to the frequent multifocality, multicentricity and bilaterality of ILC the overall extent of disease may remain underestimated.

In our study, the combination of X-ray mammography and ultrasonography correctly detected 45 of $52(86.54 \%)$ ILCs and MR - mammography alone 50 of 52 (96.15\%) ILCs, while X-ray mammography alone revealed only 25 of $52(48.08 \%)$ and ultrasonography 45 of 52 (86.54\%). In the diagnosis of ILC the most effective combination was that of $\mathrm{X}$-ray mammography and MR-mammography with sensitivity of $96.15 \%$.

Similarly in the diagnosis of IDCs the combination of Xray mammography and ultrasonography reached sensitivity of $96.5 \%$ (195 of 202), while the combination of X-ray mammography and MR - mammography correctly identified all 202 IDC lesions. The highest difference in sensitivities was confirmed at IDCs with extensive intraductal component (EIC), where the X-ray mammography found only 42 of $72(58.33 \%)$ IDCs with EIC, ultrasonography 38 of 72 (52.78\%) and MR - Mammography 65 out of $72(90.28 \%)$ IDCs with EIC. Combination of X-ray mammography and ultrasound in detection of IDC with EIC had a sensitivity of $75 \%$ and the combination of X-ray mammography and MR - mammography $100 \%(\mathrm{p}=0.0070)$.

The overall detection of focal changes in breast by MRmammography itself reached a diagnostic reliability of $72.48 \%$ (345 out of 476 lesions), X-ray mammography $69.12 \%$ (329 of 476) and ultrasonography $59.87 \%$ (285 out of 476). There was no significant difference in diagnostic confidence $(69.54 \%$ versus $69.75 \%$ ). between the combinations of X-ray mam- 
mography- ultrasonography and X-ray mammography- breast MRI in detection of total number of lesions.

These results are consistent with results of similar studies. Berg et al. [11] published higher sensitivity of MRI than mammography and ultrasound for all tumor types (depicting $95 \%$ of IDC and $96 \%$ of ILC). In their study, after combination of $\mathrm{X}$-ray mammography, clinical examination, and ultrasound, MRI identified additional tumors in another $12 \%$ of breasts. Combination of X-ray mammography, clinical examination, and MR imaging was more sensitive than any other individual test or combination of tests [11]. In comparison to this, in our study combination of X-ray mammography and MR - mammography in diagnosis of IDCs reached sensitivity of $100 \%$ and in ILC lesions $96,15 \%$. We find especially important, that the sensitivity of combined X-ray mammography and MR-mammography in IDC with EIC in our study was $100 \%$. This is essential for determination of total disease extent before planning of surgical procedure.

Overall the MR - mammography is very effective complementary and consultative modality to X-ray mammography and ultrasonography, and it is particularly important in the detection of additional, mammographically and ultrasonographically occult breast lesions, as well as in the determination of the actual extent of the pathological changes in the ipsilateral, or contralateral breast. There might be certain risk of overestimation which may lead to more radical breast surgery. The need for preoperative MR - mammography and its standard use in the preoperative diagnosis of breast cancer are widely discussed. A recent meta - analysis [13] of 21 studies demonstrated positive or resection margins less or equal to $1 \mathrm{~mm}$ for 3781 of 14,571 patients (26\%), early local recurrence for close resection margins (Odds ratio 1.8 ) and positive resection margins (Odds ratio 2.4) . In $30 \%$ of 1,648 women who underwent breast conserving surgery positive or close resection margins were found, re-excision was performed in $17 \%$ of these women, of whom $33 \%$ had residual cancer [16]. Another meta-analysis $[15,16]$ found that on the basis of MR - mammography additional breast lesions were diagnosed, what in $11 \%$ of women led to change of the scope of planned surgery on ipsilateral breast and in 3-4 \% of women contralateral breast cancer was confirmed. However, in view of the possibility of overestimation of breast changes with MR - mammography EUSOMA (European Society for Mastology) working group issued a recommendation which states indications for preoperative MR - mammography (ILC, women with known high risk for development of breast cancer, difference of $\geq 1 \mathrm{~cm}$ in average tumor size in mammography and ultrasound) [17]. In this context randomized study MONET was published [18] The study compared the number of re-operation in the group of 74 women based on preoperative MRI breast examination, and in a group of 75 women with no previous MR - mammography (34 \% vs. $12 \%$ ). Another study (COMICE), which compared the number of surgical re-excision for positive margins in two arms (MRmammography alone versus conventional breast imaging) did not find significant difference in both groups [19]. Considering the continuing controversy European Institute for Biomedical Imaging Research (EIBIR) in collaboration with the European Society of Breast Imaging (EUSOBI) are currently introducing a large two-arm multicenter prospective study (International Multicenter Prospective Meta - Analysis - MIPA). The study is aimed at comparison of the group with preoperative MR mammography and a group with conventional preoperative breast imaging [20]. The goal is an objective assessment of re-excision rates for positive resection margins and number of unilateral or bilateral mastectomies in two competing groups, as well as the percentage of changing the strategy and extent of the planned surgery, the number of local recurrences and contralateral breast cancers and appearance of distant metastases during a following 5 - years period after the primary cancer diagnosis.

We expect that as a result of further experience in accordance with existing knowledge, MR-mammography, especially its extended examination protocol (DWI, MR-spectroscopy), will retain and strengthen as a biologically most relevant method in determining the nature of breast lesions and the total extent of breast cancer. The goal remains an early and accurate diagnosis of breast lesions, especially those of malignant origin, with an adequate treatment with good cosmetic effect and quality of life with minimal risk of recurrence of the disease.

\section{Conclusion}

MR-mammography is a method of high biological relevance for the detection of additional, mammographically or ultrasonographically occult breast lesions, and for determining the actual extent of pathological changes in the ipsilateral and/or contralateral breast. Our analysis accordingly to recent studies shows that MR-mammography significantly contributes to the diagnosis of mammographically and ultrasonographically occult breast lesions, as well as to determination of the total extent of pathological changes in the breast. Therefore in cases of histologically confirmed breast cancer findings on MR-mammography should be taken into account in the planning of an effective surgical procedure.

\section{References}

1] KACL G, LIU P, DEBATIN J, GARZOLI E, CADUFF R, KRESTIN G. Detection of breast cancer with conventional mammography and contrast-enhanced MR imaging. Eur Radiol 1998; 8: 194-200. http://dx.doi.org/10.1007/ s003300050362

[2] DOGAN B, GONZALES-ANGULO A, GILCREASE M, DRYDEN M, YANG W. Multimodality imaging of triple receptor-negative tumors with mammography, ultrasound, and MRI. AJR Am J Roentgenol 2010; 194: 1160-1166.http:// dx.doi.org/10.2214/AJR.09.2355 
[3] FISCHER MA, NANZ D, HANY T, REINER CS, STOLZMANN P et al. Diagnostic accuracy of whole-body MRI/DWI image fusion for detection of malignant tumours: a comparison with PET/CT. Eur Radiol 2011;21: 246-255 http://dx.doi. org/10.1007/s00330-010-1929-X

[4] SCHELL A, ROSENKRANZ K, LEWIS PJ. Role of breast MRI in the preoperative evaluation of patients with newly diagnosed breast cancer. AJR Am J Roentgenol 2009; 192: 1438-1444 http://dx.doi.org/10.2214/AJR.08.1551

[5] EL KHOULI RH, JACOBS MA, MEZBAN SD, HUANG P, KAMEL IR et al. Diffusion-weighted imaging improves the diagnostic accuracy of conventional 3.0-T breast MR imaging. Radiology 2010; 256: 64-73. http://dx.doi.org/10.1148/ $\underline{\text { radiol.10091367 }}$

[6] KUHL C. The current status of breast MR imaging. Part I. Choice of technique, image interpretation, diagnostic accuracy, and transfer to clinical practice. Radiology 2007; 244: 356-378. http://dx.doi.org/10.1148/radiol.2442051620

[7] SCHNITT SJ, CONOLLY JL, HARRIS JR, HELMANN S, COHEN RB. Pathologic predictors of early local recurrence in stage I and II breast cancer treated by primary radiation therapy. Cancer 1984; 53:1049-1057. http://dx.doi. org/10.1002/1097-0142(19840301)53:5<1049::AIDCNCR2820530506>3.0.CO;2-O

[8] CONOLLY JL, BOYAGES J, NIXON AJ. Predictors of breast recurrence after conservative surgery and radiation therapy for invasive breast cancer. Mod Pathol 1998; 11:134-139.

[9] MANDELSON MT, OESTREICHER N, PORTER PL, WHITE D, FINDER CA et al. Breast density as a predictor of mammographic detection: comparison of interval-and screen-detected cancers. J Natl Cancer Inst 2000; 92: 1081-1087. http://dx.doi. org/10.1093/jnci/92.13.1081

[10] KOLB TM, LICHY J, NEWHOUSE JH. Comparison of the performance of screening mammography, physical examination, and breast US and evaluation of factors that influence them: an analysis of 27,825 patient evaluations. Radiology 2002. 225: 165-175. http://dx.doi.org/10.1148/radiol.2251011667

[11] BERG WA, GUTIERREZ L, NESS-AIVER MS, CARTER WB, BHARGAVAN $M$ et al. Diagnostic accuracy of mammography, clinical examination, US, and MR imaging in preoperative assessment of breast cancer. Radiology. 2004. 233: 830-849. http://dx.doi.org/10.1148/radiol.2333031484

[12] SARDANELLI F, GIUSEPPETTI GM, PANOZZA P, BAZZOCCHI M, FAUSTO A et al. Sensitivity of MRI Versus Mammography for Detecting Foci of Multifocal, Multicentric Breast Cancer in Fatty and Dense Breasts Using the Whole-
Breast Pathologic Examination as a Gold Standard. AJR Am J Roentgenol 2004. 183:1149-1157.http://dx.doi.org/10.2214/ ajr.183.4.1831149

[13] HOUSSAMI N, MACASKILL P, MARINOVICH ML, DIXON JM, IRWIG L et al. Meta-analysis of the impact of surgical margins on local recurrence in women with earlystage invasive breast cancer treated with breast-conserving therapy. Eur J Cancer. 2010, 46: 3219-3232. http://dx.doi. org/10.1016/j.ejca.2010.07.043

[14] KURNIAWAN ED, WONG MH, WINDLE I, ROSE A, MOU A et al. Predictors of surgical margin status in breastconserving surgery within a breast screening program. Ann Surg Oncol. 2008, 15: 2542-2549. http://dx.doi.org/10.1245/ s10434-008-0054-4

[15] HOUSSAMI N, CIATTO S, MACASKILL P, LORD SJ, WARREN RM et al. Accuracy and surgical impact of magnetic resonance imaging in breast cancer staging: systematic review and meta-analysis in detection of multifocal and multicentric cancer. J Clin Oncol. 2008, 26: 3248-3258. http://dx.doi. org/10.1200/JCO.2007.15.2108

[16] BRENNAN ME, HOUSSAMI N, LORD S, MACASKILL P, IRWING L et al. Magnetic resonance imaging screening of the contralateral breast in women with newly diagnosed breast cancer: systematic review and meta-analysis of incremental cancer detection and impact on surgical management. J Clin Oncol. 2009, 27: 5640-5649. http://dx.doi.org/10.1200/ LCO.2008.21.5756

[17] SARDANELLI F, BOETES C, BORISCH B, DECKER T, FEDERICO $\mathrm{M}$ et al. Magnetic resonance imaging of the breast: recommendations from the EUSOMA working group. Eur J Cancer. 2010. 46: 1296-1316. http://dx.doi.org/10.1016/j. ejca.2010.02.015

[18] PETERS NH, VAN ESSER S, VAN DEN BOSCH MA, STORM RK, PLAISIER PW et al. Preoperative MRI and surgical management in patients with nonpalpable breast cancer: the MONET - randomised controlled trial. Eur J Cancer. 2011. 47: 879-886. http://dx.doi.org/10.1016/j.ejca.2010.11.035

[19] TURNBULL L, BROWN S, HARVEY I, OLIVIER C, DREW $\mathrm{P}$, et al. Comparative effectiveness of MRI in breast cancer (COMICE) trial: a randomised controlled trial. Lancet. 2010; 375: 563-571. http://dx.doi.org/10.1016/S0140-6736(09)62070-5

[20] Preoperative Breast MRI in Clinical Practice: Multicenter International Prospective Meta-Analysis (MIPA) of Individual Woman Data An EIBIR-EuroAIM/EUSOBI Study. 2012; $<$ http://www.eusobi.org/html/img/pool/MIPA_Outline.pdf $>$. 